\title{
ELECTROMAGNETIC SELF-EXCITATION EFFECTS \\ IN \\ LIQUID METAL COOLED REACTORS
}

by

D. L. Lessor

Mathematics and Physics Research Section

Physics and Instrumentation Department

December 1973

This report was prepared as an account of work sponsored by the United States Government. Neither the United States nor the United States Atomic Energy Commission, nor any of their employees, nor any of their contractors, subcontractors, or their employees, makes any warranty, express or implied, or assumes any legal liability or responsibility for the accuracy, completeness or usefulness of any information, apparatus, product or process disclosed, or represents that its use would not infringe privately owned rights.

BATTELLE

PACIFIC NORTHWEST LABORATORIES

RICHLAND, WASHINGTON

Work performed for the U. S. Atomic Energy Commission under work order C28159 from Westinghouse Hanford Corporation 


\section{DISCLAIMER}

This report was prepared as an account of work sponsored by an agency of the United States Government. Neither the United States Government nor any agency Thereof, nor any of their employees, makes any warranty, express or implied, or assumes any legal liability or responsibility for the accuracy, completeness, or usefulness of any information, apparatus, product, or process disclosed, or represents that its use would not infringe privately owned rights. Reference herein to any specific commercial product, process, or service by trade name, trademark, manufacturer, or otherwise does not necessarily constitute or imply its endorsement, recommendation, or favoring by the United States Government or any agency thereof. The views and opinions of authors expressed herein do not necessarily state or reflect those of the United States Government or any agency thereof. 


\section{DISCLAIMER}

Portions of this document may be illegible in electronic image products. Images are produced from the best available original document. 


\section{TABLE OF CONTENTS}

Introduction and Summary

Elementary Model Exhibiting Self-Excitation

General Necessary Conditions for Self-Excitation

Application of Magnetic Reynolds Number Criterion to FFTF

Possible Electromagnetic Self-Excitation Geometries in LMFBR'S

1. Reactor Core Possibilities

2. Centrifugal Pump Region Self-Excitation Possibilities

3. Other Radial Disk Flows

Appendix 1. Electromagnetic Self-Excitation in Cylindrical Radial Flows

\section{LIST OF FIGURES}

Figure 1 - Homopolar generator with self-excitation feedback.

Figure 2 - Current flow tendency in reactor core with horizontal magnetic fleld.

Figure 3 - Path needed to give self-excitation phenomena in reactor core.

Figure 4 - Effect of azimuthal velocity and magnetic field component perpendicular to centrifugal pump axis.

Figure 5 - Effect of azimuthal velocity and magnetic field component parallel to impeller axis.

Figure 6 - Current flow $\underline{\mathrm{J}}$ and induced magnetic field from radial outward flow and magnetic field component parallel to pump axis.

Figure 7 - Current flow $\underline{J}$ and induced magnetic field from radial inward flow and magnetic field component parallel to pump axis. 


\section{ELECTROMAGNETIC SELF-EXCITATION EFFECTS}

in

LIQUID METAL COOLED REACTORS

D. L. Lessor

\section{Introduction and Summary}

Electromagnetic self-excitation effect is a possible problem in LMFBR's. Geometry and flow parameters may rule it out; the magnetic Reynolds number criterion does not. The notes with this memo treat components in which it may occur in liquid metal loops, show certain required conditions for its occurrence, and give some idea of its consequences.

The electromagnetic self-excitation effect is the phenomenon in which conducting material moves in a magnetic field in such a way that the resulting emf (electromotive force) drives a current which in turn produces a magnetic field which enhances the original. It has been suggested by M. K. Bevir* that self-excitation may occur accidentally in liquid metal cooled reactors.

If this phenomenon occurred in a liquid metal flow system, the result would be onset of an anomalous pressure drop at some threshold velocity and large electric currents in the liquid metal flow system. Pumping power could be increased dramatically after onset of the effect with negligible increase in flow velocity in the coolant loop element in which the effect occurred. Electric currents and magnetic fields would build to some level determined by power driving the flow.

Criteria for the occurrence of this phenomenon are:

(1) magnetic Reynolds number greater than one,

(2) geometry favorable to magnetic field reinforcement.

\footnotetext{
*M. K. Bevir, "Possibility of Electromagnetic Self-Excitation in Liquid Metal Flows in Fast Reactors," J. Br. Nucl. 12, 455 (1973)
} 
The magnetic Reynolds number is discussed in the accompanying notes and is found to be much larger than unity in components of the FFTF. Hence an examination of geometry is needed.

A core region with vertical upward flow in the Northern hemisphere would have a predilection for self-excitation if large current paths carrying current over the top of the vertical flow region from the magnetic east to the magnetic west side were much more favorable than current paths under or around the collection of flow ducts.

A centrifugal pump could exhibit self-excitation from a magnetic field component parallel to its axis and the azimulthal (rotational) velocity of the fluid and impeller, provided an external electrical circuit path carries current between impeller shaft region and impeller tip region with a preferred helicity of the correct sign around the impeller axis. Usual pump design would usually have negligible looping about the impeller axis in such paths, or the two helicities of such looping would be equally preferred.

The radial outward motion of the fluid in a centrifugal pump will not give a self-excitation effect with a magnetic field component parallel to its axis, but a radial inward flow can give the effect very dramatically.

A disk of radial inward conducting fluid flow with a magnetic field normal to the plane of the disk appears to be the most likely situation for accidental self-excitation. An example would be a near empty sodium tank being drained toward a center exit duct. A numerical example is treated in the accompanying notes. The effect on pressure drops for this case is sila 11 , even though the electric currents are large.

The major shortcoming of the analysis used in the radial disk flow model, for which the mathematical development appears as an appendix, is the assumed time independence. While it shows what conditions would occur in one steady state solution, it does not prove that that is the solution which develops from real initial conditions. The solution exhibited may give small effects because its magnitude is set by the assumption of magnetic field equal to the ambient (taken as earth's or component thereof) at the 
outer flow boundary. The elementary model exhibiting self-excitation discussed in the next section shows that if magnetic Reynolds number is sufficiently high, a field and current buildup can be initiated by a transient field.

We recommend that subsequent work treat time dependence of selfexcitation effects in liquid metal flow systems.

\section{Elementary Model Exhibiting Self-Excitation}

The essential feature of electromagnetic self-excitation is this: Current flowing as a result of conductor moving in a magnetic field increases that magnetic field. Result: Current increases, field increases, and/or motion is restricted. Work must be done to drive this current; hence there is no "runaway" phenomenon. Resuiting forces on the conductor will try to oppose motion.

A homopolar generator can be wired to exhibit self-excitation, and it also is an idealization of some potential self-excitation systems that could occur in a liquid metal flow system. An idealized homopolar generator with self-excitation feedback is shown in Figure 1.

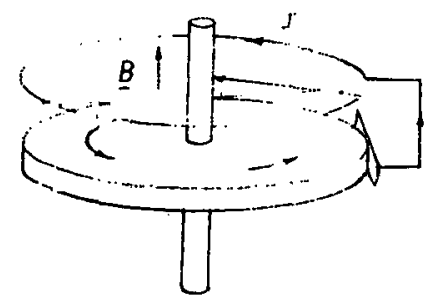

Figure 1

Homopolar Generator With Self-Excitation Feedback 
The electromotive force (emf) produced by the generator rotating with angular velocity $\omega$ is

$$
\varepsilon=1 / 2 \omega B_{t}\left(r_{2}^{2}-r_{1}{ }^{2}\right)
$$

where $r_{2}$ and $r_{1}$ are inner and outer radii and $B_{t}$ is the total magnetic field component normal to the plane of the disk. We can separate $B_{t}$ into a part $B_{0}$ present from external sources and a part $B=\alpha I$ proportional to current in the loop.

$$
\begin{aligned}
B_{t} & =B_{0}+B \\
& =B_{0}+\alpha I
\end{aligned}
$$

Here $\alpha$ is a proportionality constant describing the feedback.

The differential equation for current $I$ in the loop of resistance $R$ and self-inductance $L$ may be written

$$
\begin{aligned}
L \frac{d I}{d t}+I R & =\varepsilon \\
& =1 / 2 \omega\left(r_{2}{ }^{2}-r_{1}{ }^{2}\right) B_{t} \\
& =\beta \omega\left(B_{0}+\alpha I\right)
\end{aligned}
$$

where $\beta=\left(r_{2}^{2}-r_{1}^{2}\right) / 2$.

If the current in the loop makes $\mathrm{N}$ turn in a plane circle close to the plane of the disk with approximately the same radius $r_{2}$ as the disk, the magnetic field at the center will be approximately

$$
B=\frac{\mu_{0} N I}{2 r_{2}} \text {. }
$$

Allowing some leakage flux, we will use this as an approximate value throughout the plane of the disk. Hence we have

$$
\alpha=\frac{\mu_{0} N}{2 r_{2}} \text {. }
$$

Starting from an initial current zero, the solution to the differential equation (5) is 


$$
I=\frac{\beta \omega B_{0}}{R\left(1-\frac{\beta \omega \alpha}{R}\right)}\left(1-e^{-\frac{R}{L}\left(1-\frac{\beta \omega \alpha}{R}\right) t}\right) .
$$

Equation (8) says that current approaches a steady state value

$$
I(t=\infty)=\frac{\beta \omega B_{0}}{R\left(1-\frac{\beta \omega \alpha}{R}\right)}
$$

unless the exponent is positive:

$$
-\frac{R}{L}\left(1-\frac{\beta \omega \alpha}{R}\right)>0
$$

or

$$
\frac{\beta \omega \alpha}{R}>1 \text {. }
$$

Positive exponent implies fields and currents buildup. In practice, of course, the current does not become infinite as would be inferred from equation (8) because an infinite torque would be required to maintain constant angular velocity $\omega$.

If we take $r_{2}>>1$, the criterion (11) for self-excitation in this homopolar generator is

$$
\frac{\beta \omega \alpha}{R}=1 / 2 r_{2}^{2} \omega\left(\frac{\mu_{0} N}{2 r_{2}}\right) / R>1 .
$$

Suppose that the electrical resistance $R$ comes from the $N$ turns of conductor looping around the disk:

$$
R \simeq \frac{\ell}{A \sigma}=\frac{2 \pi r_{2} N}{\sigma A}
$$

The criterion (12) becomes

$$
\frac{\beta \omega \alpha}{R} \simeq 1 / 2 \mu_{0} \underbrace{\left(\omega r_{2}\right)}_{\text {Maximum velocity }} \sigma \underbrace{(\underbrace{\frac{A}{2 \pi r_{2}}})>1}_{\begin{array}{l}
\text { Conductor area/path length, dimensionally a } \\
\text { distance }
\end{array}}>14
$$

The criterion (14) is informative in that it can be used to estimate areas 
of conducting paths needed to give an accidental self-excitation hazard in sodium loop elements. Criterion (14) fits the form of a criterion which we shall arrive at with vaguer but more general arquments.

Note in this analysis the current at any instant of time (equation 8) or in steady state (equation 9) is proportional to the externally applied magnetic field $B_{0}$. It would perhaps be useful in follow-on work to consider a transient external field as starting the accidental self-excitation. In subsequent analysis in this report we will consider only steady state conditions, even though this may miss some important effects. The examination of time dependent effects in liquid metal flow systems is recommended.

To illustrate the starting of self-excitation by a transient field, suppose the externally applied magnetic field is $B_{0}$ up to time $t_{1}$ and is zero thereafter for our idealized homopolar generator. For time $t>t_{1}$, the current equation instead of equation ( 8 ) would be

$$
I=\frac{\beta \omega B_{0}}{R\left(1-\frac{\beta \omega \alpha}{R}\right)}\left(1-e^{-\frac{R}{L}\left(1-\frac{\beta \omega \alpha}{R}\right) t} 1\right) e^{-\frac{R}{L}\left(1-\frac{\beta \omega \alpha}{R}\right)\left(t-t_{1}\right)} .
$$

This shows that the current could continue to build up after the externally applied field had disappeared.

General Necessary Conditions for Self-Excitation

The magnetic Reynolds number criterion for possible self-excitation can be arrived at from ohm's law and one of the Maxwell equations (Faraday's Law) in integral or differential form. Faraday's Law says that electric current density $\underline{\mathrm{J}}$ serves as a magnetic field source according to

$$
\nabla \times \underline{B}=\mu_{0} \underline{J}
$$

or

$$
\oint_{C} \underline{B} \bullet \underline{d l}=\iint_{s} \mu_{0} \underline{J} \bullet \underline{d s} .
$$

Here $s$ is a surface and $c$ is its boundary. Ohm's law for a conducting material medium moving with velocity $\underline{u}$ states

$$
\underline{\mathrm{J}}=\sigma(\underline{\mathrm{E}}+\underline{\mathrm{u} X} \mathrm{~B})
$$


where $\sigma$ is electrical conductivity. Hence magnetic field contribution to the current density can be of the order

$$
J=\sigma u B
$$

where $u$ and $B$ are mutually perpendicular components of $\underline{u}$ and $\underline{B}$. Suppose a mathematical surface $s$ with boundary $c$ exists such that a current density $\underline{J}$ of order of magnitude ouB ${ }_{\text {old }}$ flows through the surface, in addition to possible current flowing due to electric field. This current serves as a source of a new magnetic field $B_{\text {new }}$ which exists along some distance $L$ of the boundary $c$. From equation (17) we conclude

or

$$
\operatorname{Max}\left(B_{\text {new }} L\right)=\operatorname{Order}\left(\mu_{0} \sigma u B_{o l d} A\right)
$$

$$
\operatorname{Max} B_{\text {new }}=\operatorname{Order}\left(\mu_{0} \sigma u A / L\right) * B_{0 l d} .
$$

A necessary condition for self-excitation is that the resulting magnetic field $B_{\text {new }}$ be of the same order as the field $B_{\text {old }}$, flow transverse to which results in electrical current:

$$
\mu_{0} \sigma \cup A / L \gtrsim 1 \text {. }
$$

If in fact the geometry is such that the resulting field exists at the same location as that which caused the current flow and is parallel to it, the self-excitation phenomenon can occur. In the criterion (22), the ratio $A / L$ is usually written simply as some typical linear dimension $\ell$ and the resulting dimensionless number is called the magnetic Reynolds number:

$$
R_{m}=\mu_{o} \text { oul } \text {. }
$$

Application of Magnetic Reynolds Number Criteria to FFTF

Consider the magnetic Reynolds number for the assembly of vertical flow ducts through core. For the magnetic Reynolds number test we use

$$
\begin{aligned}
& \mu_{0}=4 \pi \times 10^{-7} \\
& \sigma=3.42 \times 10^{6} \text { (olun meter) }^{-1} \\
& u=6.7 \text { meters } / \mathrm{sec}
\end{aligned}
$$

Sodium conductivity, corresponding to resistivity 11.5 micro ohm inch at $550{ }^{\circ} \mathrm{C}$

Flow velocity, about $22 \mathrm{ft} / \mathrm{sec}$ 


$$
\ell=2.5 \text { meters }
$$

The values (24) yield

$$
R_{m}=72 \text {. }
$$

The necessary condition for self-excitation is met. One must next look for possible geometric configurations within the sodium flow where electromagnetic self-excitation can occur.

\section{Possible Electromagnetic Self-Excitation Geometrics in LMFBR's}

The essential features of a self-excitation geometry are

(1) flow of conducting material in a direction normal to a magnetic field component,

(2) a current path in the right orientation and sense to enhance the magnetic field inducing the current.

\section{Reactor Core Possibilities}

To give self-excitation in the reactor core with liquid metal flowing upward, one would need a current path over or under the reactor (depending on location). Current paths with the wrong orientation seem equally likely. An LMFBR in washington with upward flow through core should have a preferred current path running from the magnetic east side of the reactor over the top to the magnetic west side to exhibit self-excitation in the core. A current loop running from the magnetic east side of the reactor core under the redictor to the magnetic west side opposes the effect from the earth's magnetic field. See Figure 2 and Figure 3. Current whose path outside the core merely runs around the core barrel does not contribute significantily to the effect. The area of the current path over the top of the core must be large (and the flow area around the sides and under the bottom would need to be small) for the electromagnetic self-excitation effect to occur. This could possibly occur in certain swimming pool type LMFBR's. To see what order of magnitude current path is needed, consider a vertical liquid metal flow stream of thickness $\ell$ and vertical length $s$ flowing with velocity $v$. The magnetic induced emf from the earth's horizontal magnetic field will be approximately 


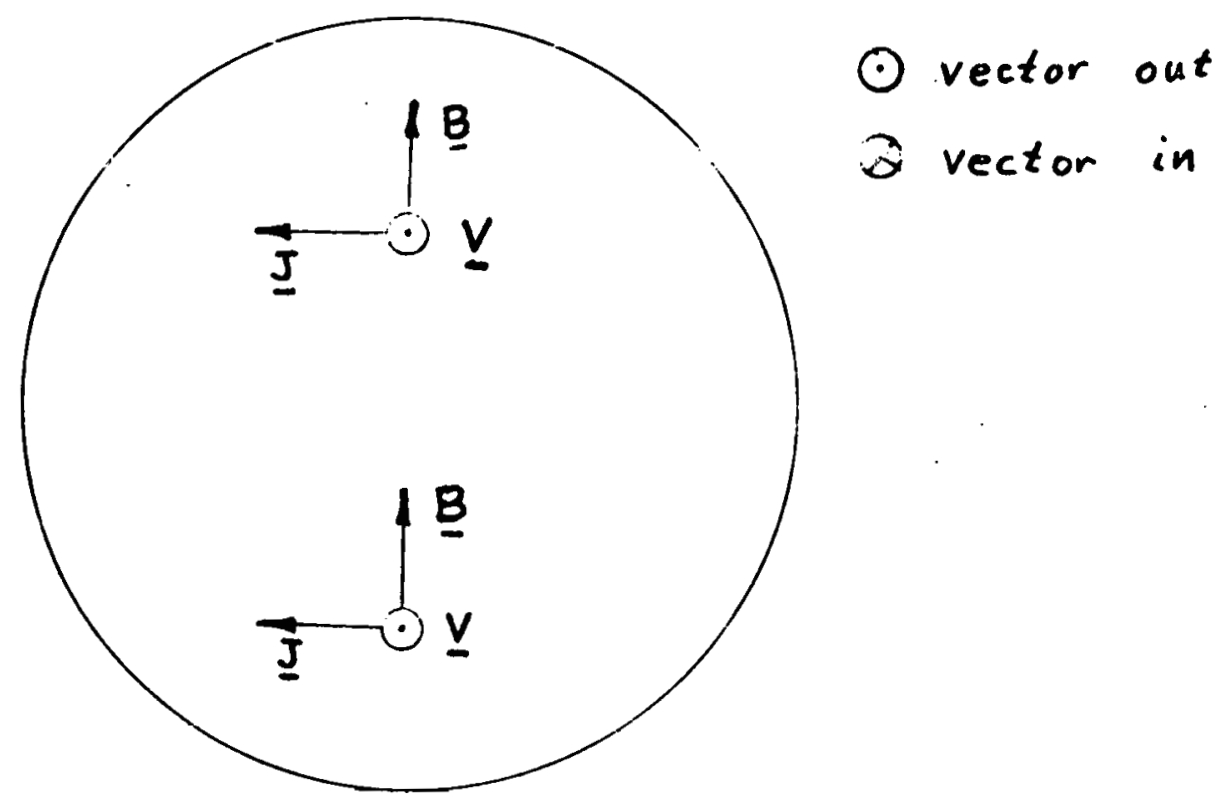

Fig. 2 Current flow tendency in reactor core with horizontal magnetic field

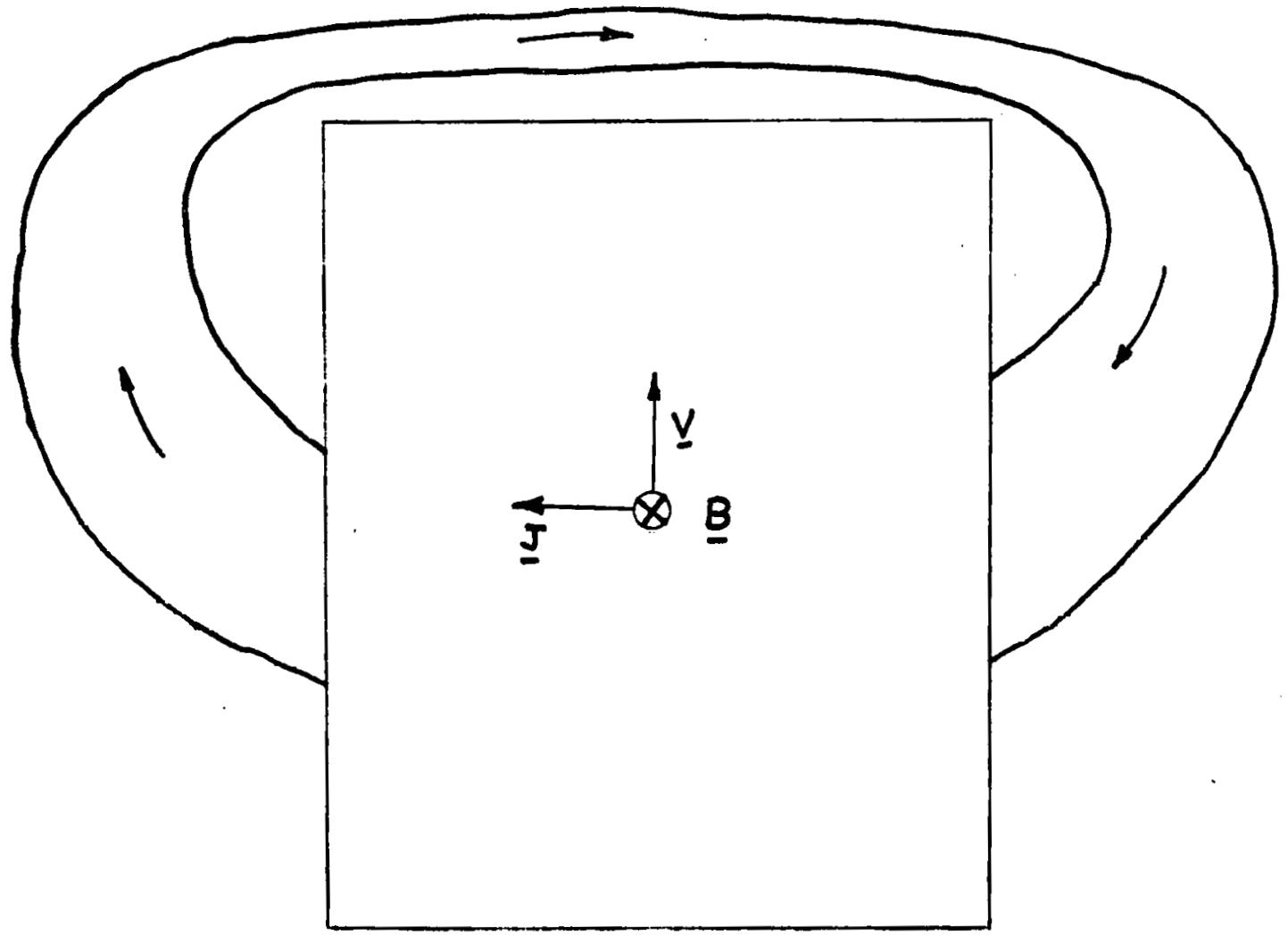

Fig. 3 Path needed to give self-excitation phenomena in reactor core $-9-$ 


$$
\varepsilon=\mathrm{B} \vee \ell,
$$

where $B$ is eath's magnetic induction horizontal component. Consider an external approximately circular current path of radius $s$ and cross sectional area $A$. The current carried will be of the order

$$
I=\frac{\varepsilon}{R}=\frac{\varepsilon}{\left(\frac{2 \pi S}{\sigma A}\right)}=\frac{B v \ell}{\left(\frac{2 \pi S}{\sigma A}\right)} \text {. }
$$

The average new magnetic field felt by the flowing liquid metal as a result of this current will be of the order

$$
B_{\text {new }}=\frac{\mu_{0} I}{s}=\frac{\mu_{0} v \sigma l A}{2 \pi s^{2}} B \text {. }
$$

Take $v=6.7$ meter $/ \mathrm{sec}(\sim 22 \mathrm{ft} / \mathrm{sec}), s=3$ meter, $\ell=1$ meter, $\sigma=$ $3.42 \times 16^{6} \mathrm{ohm}^{-1}$ meter ${ }^{-1}$ (hot sodium conductivity). Requiring

$$
\mu_{0} v \sigma \cdot \frac{l A}{2 \pi s^{2}}=1
$$

requires

$$
\mathrm{A} \simeq .65 \operatorname{meter}^{2} \simeq 7 . \mathrm{ft}^{2}
$$

A current path of this size in sodium would be required, and current flow under or around the core would need to be suppressed for electromagnetic self-excitation to occur in the reactor core region.

\section{Centrifugal Pump Region Self-Excitation Possibilities}

Because of the high speed flow in it, the centrifugal pump is a candidate fon electromagnetic self-excitation phenomena. To examine the current flow tendencies, let us break the flow velocity into azimuthal and radial components, and the magnetic field into components parallel and perpendicular to the axis of the pump impeller. We will then consider the interaction of these velocity and field components pairwise.

Figure 4 shows the direction of current density $\mathrm{J}$ from the magnetic field component perpendicular to the pump axis and the azimuthal velocity. The resulting eddy currents might cause Galvanic erosion of pump surfaces but do not with ordinary pump geometry contribute to self-excitation. 


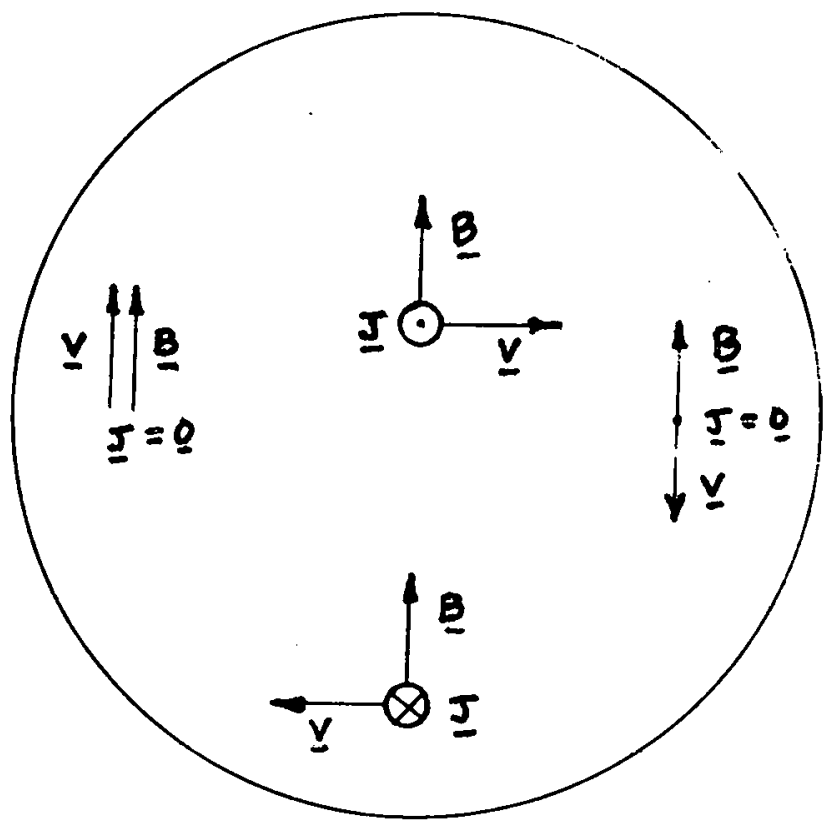

$\odot$ vector out

$\otimes$ vector in

Fig. 4 Effect of azimuthal velocity and magnetic field component perpendicular to centrifugal pump axis

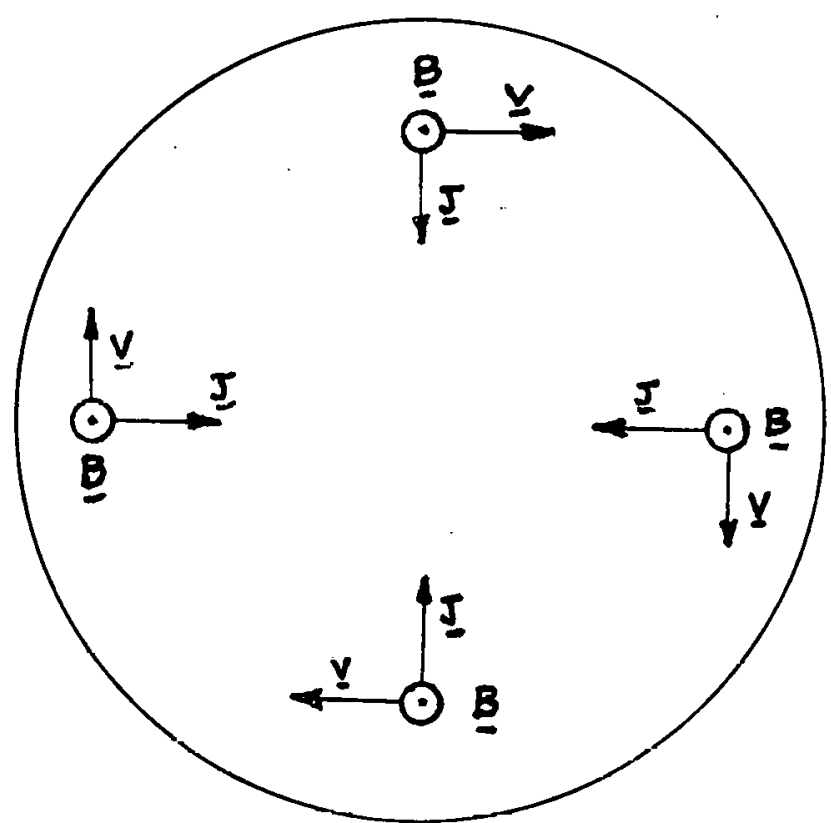

Fig. 5 Effect of azimuthal velocity and magnetic field component parallel to impeller axis 
Figure 5 shows the direction of electrical current density $\underline{J}$ flowing as a result of azimuthal velocity and a magnetic field component parallel to the pump axis. This resembles a homopolar generator. The current flow within the fluid tends to be outward or inward according to whether $\underline{B}$ is parallel or antiparallel to the rotational angular velocity. (Antiparallel is shown in Figure 5 ; parallel is shown in Figure 1). Selfexcitation is favored if current flow external to the rotating fluid and impeller tends to loop about the axis of rotation in the favorable direction in going between impeller axis and rotating fluid periphery. The favorable direction of such looping of the current is parallel to the peripheral fluid velocity if rotation angular velocity vector is parallel to $\underline{B}$, and antiparallel to the peripheral fluid velocity if rotation angular velocity is antiparallel to $\underline{B}$. A preferred helicity in an external current path between pump axis and impeller periphery should be avoided if self-excitation is to be avoided. There seems to be no obvious reason for introducing such helicity in current paths. The homopolar generator effect will occur in the impeller with whatever fields are present, but they probably will not self-excite.

Radial outward flow as in a centrifugal pump generates a current which gives rise to a magnetic field which has the opposite sense to the axial component causing the emf. See Figure 6 . Hence no flux enhancement or selfexcitation occurs.

Radial inward flow, by contrast, generates a current whose resulting magnetic field enhances that initially present. See Figure 7 . An increased pressure drop from enhanced magnetic field will occur radially. The current and field will grow to some value determined by pump power and limiting pump head. For radial flow of incompressible liquid of conductivity $\sigma$ in an annular region of outer radius $r_{1}$, it can be shown that the pressure at radius $r<r_{1}$ is given in steady state by

$$
p=p_{1}+\frac{B_{1}^{2}}{2 \mu_{0}}\left[1-\left(\frac{r}{r_{1}}\right)^{2 \mu_{0} \sigma u_{1} r_{1}}\right]+\frac{1}{2 p}\left(\frac{\dot{m}}{2 \pi}\right)^{2}\left(\frac{1}{r_{1}}-\frac{1}{r^{2}}\right) .
$$

Here $p_{1}$ is pressure at outer radius $r_{1}, u_{1}$ is the radial velocity of the 


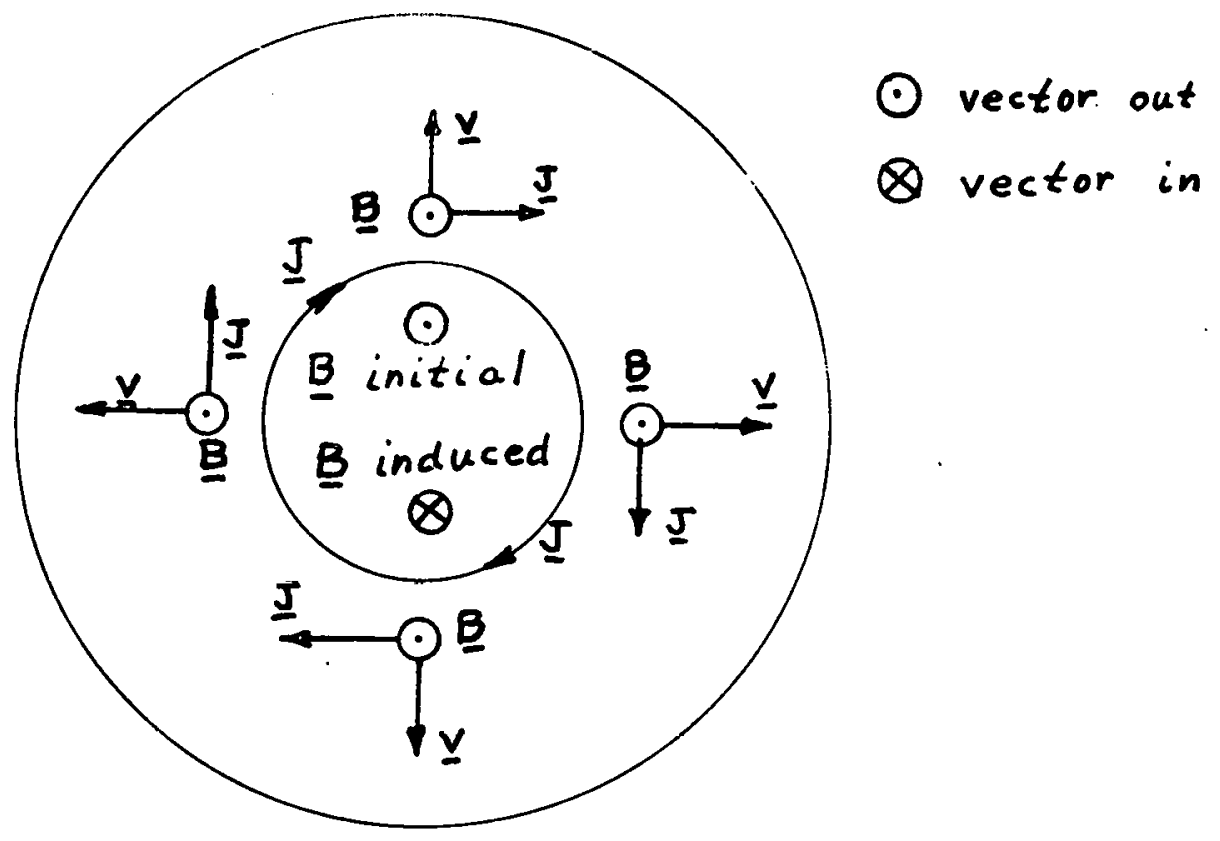

Fig. 6 Current flow $\mathrm{J}$ and induced magnetic field from radial outward flow and magnetic field component parallel to pump axis

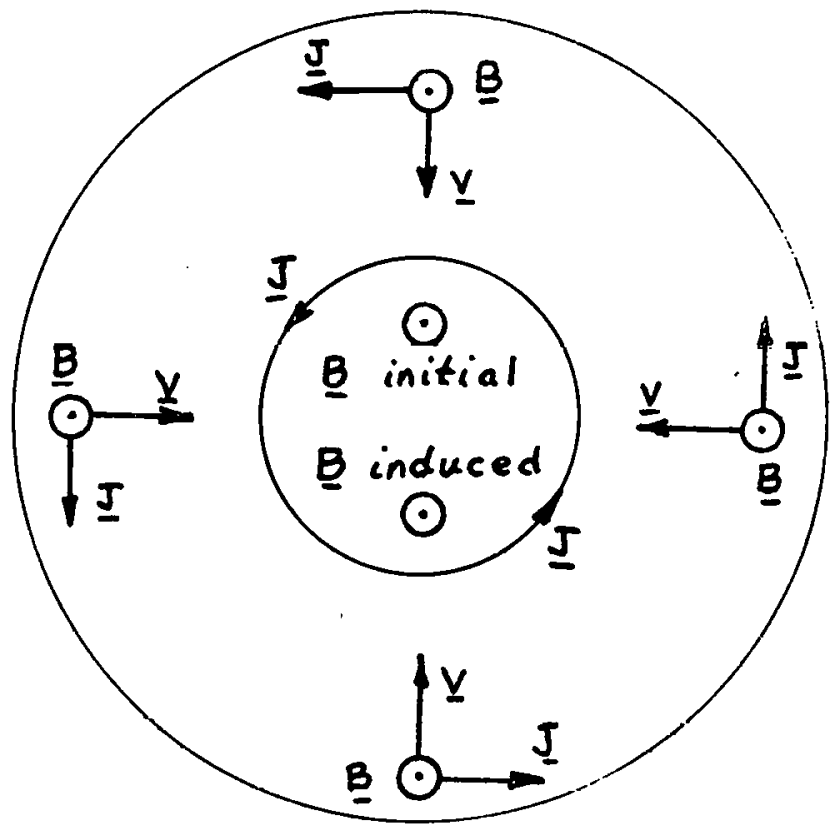

Fig. 7 Current flow $\mathrm{J}$ and induced magnetic field from radial inward flow and magnetic field component parallel to pump axis 
fluid at outer radius $r_{\eta}, \rho$ is fluid density, and $m$ is the radial mass flow rate per unit cylinder length. The right hand term is like a Bernoulli pressure drop and is independent of magnetic field. Rationalized MKS units are implied by the presence of the $\mu_{0}$. The pressure term proportional to the axial magnetic field $B_{1}$ at outer radius $r_{1}$ is a magnetic pressure drop term:

$$
\left(p-p_{1}\right)_{M a g}=\frac{B_{1}^{2}}{2 \mu_{0}}\left[1-\left(\frac{r}{r_{1}}\right)^{2 \mu_{0} \sigma u_{1} r_{1}}\right] \text {. }
$$

Note that for outward flow $\left(u_{1}>0\right),\left(p(r)-p\left(r_{1}\right)\right)$ magnetic is negative and can be of large magnitude if $\mu_{0} \sigma u_{1} r_{1}>1$. Thus a large pressure on the outside would be necessary to force flow inward.

Since backflow through the pump is not anticipated, we should look elsewhere in the system for radial inward disk flow. We note, however, that if one attempted to backflow through a 2-1/4 inch thick disk shaped annulus of outer diameter 40 inches and inner diameter 18 inches a volume flow rate of 18,000 gallons/minute of sodium, the pressure required to do so at most unfavorable orientation in Washington State would be $500 \mathrm{lbs} / \mathrm{in}^{2}$ due to self-excitation effects alone. These dimensions (except for inner pipe diameter) and flow rates are typical of the FFTF primary pump in normal forward flow. The 2-1/4 inch thickness is a slot width at impeller tip. Reducing the inner radius or increasing the flow rate would further increase the magnitude of the required pressure, while orienting the axis at some angle away from parallel to the earth's maynelic field would roduce it.

\section{Other Radial Disk Flows}

The radial inward disk flow geometry could occur in the flow from a liquid metal storage tank out through a central orifice. The geometric assumptions of the idealized model are best met in an almost empty cylindrical tailk. Consider a tank in which flow is radially inward from a radius of 10 feet to a radius of .75 feet. Suppose al so that one foot depth of liquid metal remains in the tank. The assumptions of our mathematical model are not strictly met; for example, flow velocity is not strictly proportional to $r^{-1}$ and we do not. have invariance in the cylinder axis direction. 
Nevertheless, our model provides an estimate of the radial pressure drop due to the vertical component of the earth's magnetic field:

$$
\begin{aligned}
& \frac{r}{r_{1}}=\frac{.75}{10}=.075 \\
& 2 \pi r_{1} u_{1} L=15,000 \frac{\text { gal }}{\min } \\
& =.946 \operatorname{meter}^{3} / \mathrm{sec} \\
& \text { Volume flow rate } \\
& \sigma=3.42 \times 10^{6} \text { (ohm meter) }^{-1} \\
& L=1 \text { foot }=.3048 \text { meter Depth of sodium } \\
& r_{1}=10 \text { feet }=3.048 \text { meter Outer radius } \\
& B_{1}=.55 \times 10^{-4} \text { tesla Vertical component of } \\
& \text { earth's magnetic field } \\
& \text { in Washington } \\
& \left(p-p_{1}\right)_{\text {Mag }}=\frac{B_{1}^{2}}{2 \mu_{0}}\left(1-\left(\frac{r}{r_{1}}\right)^{2 \mu_{0} \sigma r_{1} u_{1}}\right) \\
& =-72.6 \mathrm{nt} / \mathrm{m}^{2} \\
& =-1.05 \times 10^{-2} \text { pound } / \mathrm{in}^{2} \text {. }
\end{aligned}
$$

If this flow came in to a tube of inner radius 6 inches instead of 9 inches, the pressure drop would be

$$
\left(p-p_{1}\right)_{\text {Mag }}=-406 . n t / m^{2}=-.05 \text { pound } / \mathrm{in}^{2} \text {. }
$$

Note that we have assumed that the axial magnetic field present at the outer periphery for the draining tank case was the vertical component of the earth's magnetic field. This is probably not a bad assumption for our draining tank example. For the centrifugal pump backflow estimate, the magnetic field at the periphery was taken as the total magnetic field of the earth in the state of Washington; that is, the worst possible pump orientation was chosen. The analysis, of course, assumed no ferromagnetic material present in the vicinity of the flowing conductors. The presence of ferromagnetic flux loops could increase magnetic flux reinforcement and make the magnitude 
of the earth's magnetic field irrelevant except for starting the magnetic field buildup transient.

The magnetic field present at any liquid metal flow system element, may, of course, be dramatically lower than that of the earth because of shielding by structural steel in building structures. 


\section{Appendix 1}

\section{Electromagnetic Self-Excitation in Cylindrical Radial Flows}

The equations governing the steady state flow of an incompressible conducting fluid in a steady electromagnetic field are:

Continuity of mass:

$$
\nabla \cdot \underline{u}=0
$$

Momentum Balance

$$
\left.\rho \underline{u} \bullet \nabla \underline{u}=-\nabla p+v \rho\left[\nabla^{2} \underline{u}+1 / 3 \nabla \nabla \cdot \underline{u}\right)\right]+\underline{J} \times \underline{B}
$$

Ohm's Law

$$
\underline{J}=\sigma(\underline{E}+\underline{u} \times \underline{B})
$$

Maxwell's Equations

$$
\begin{array}{rll}
\nabla X \underline{E}=0 & (4) & \nabla \bullet E=0 \\
\nabla X \underline{B}=\mu_{0} \underline{J} & (5) & \nabla \bullet \underline{B}=0
\end{array}
$$

Here $\underline{u}$ is fluid velocity, $\nu$ is kinematic viscosity, $\rho$ is fluid density, $\sigma$ is fluid conductivity, $\underline{J}$ is electric current density, $\underline{E}$ and $\underline{B}$ are electric and magnetic induction fields, and $\mu_{0}$ is the rationalized MKS permeability of free space.

Let us assume radial flow in cylindrical symmetry with no $Z$ (axial) dependence. Let us also assume only an axial magnetic field is present. Then we have from equation (5)

$$
\begin{aligned}
\underline{J} \underline{X} \underline{B} & =\frac{1}{\mu_{0}}(\nabla X \underline{B}) \times \underline{B} \\
& =-\nabla\left(\frac{\underline{B}^{2}}{2 \mu_{0}}\right)+\frac{1}{\mu_{0}} \underline{B} \cdot \nabla \underline{B} \\
& =-\nabla\left(\frac{B^{2}}{2 \mu_{0}}\right) .
\end{aligned}
$$

The $\underline{B} \bullet \underline{B}$ term vanishes for $\underline{B}=B(r) \hat{Z}$, since for that case

$$
\underline{B} \bullet \nabla \underline{B}=B \frac{\partial}{\partial Z} \quad B(r) \widehat{Z} \text {. }
$$


The momentum balance radial equation becomes

$$
u \frac{d u}{d r}=-\frac{1}{\rho} \frac{d p}{d r}+v\left(\frac{d^{2} u}{d r^{2}}+\frac{1}{r} \frac{d u}{d r}-\frac{u}{r^{2}}\right)-\frac{1}{\rho} \frac{d}{d r}\left(\frac{B^{2}}{2 \mu_{0}}\right)
$$

or

$$
\frac{d}{d r}\left(\frac{1}{2} \cdot u^{2}\right)=-\frac{1}{\rho} \frac{d}{d r}\left(p+\frac{B^{2}}{2 \mu_{0}}\right)+v\left(\frac{d^{2} u}{d r^{2}}+\frac{d}{d r}\left(\frac{u}{r}\right)\right) .
$$

Hence integrating gives

$$
\frac{1}{2} u^{2}+\frac{1}{\rho}\left(p+\frac{B^{2}}{2 \mu_{0}}\right)+v\left(\frac{d u}{d r}+\frac{u}{r}\right)=k_{1} .
$$

For a purely radial flow with $\underline{u}=u(r) \hat{r}$, mass continuity implies

$$
\nabla \cdot \underline{u}=\frac{d u}{d r}+\frac{u}{r}=0
$$

or

$$
\begin{aligned}
u & =k_{2} / r \\
& =\frac{\dot{m}}{2 \pi r \rho}=u_{1} \frac{r_{1}}{r} .
\end{aligned}
$$

where $\dot{m}$ is radial mass flow rate per unit length, positive outward. Hence we have

$$
\frac{1}{2} u^{2}+\frac{1}{\rho}\left(p+\frac{B^{2}}{2 \mu_{0}}\right)=k_{1}
$$

with flow velocity $u$ given by equation (17).

To give the pressure drop between two radii we still need the radial magnctic field dependence in equation (18). Taking the curl of equation (3) and using equation (4) and equation (5) gives

$$
\sigma \nabla X(\underline{u} \times \underline{B})=\frac{1}{\mu_{0}} \nabla \times(\nabla \times \underline{B})
$$

or

$$
\nabla \times\left(\underline{\text { UXX }} \underline{\mathrm{B}}-\frac{1}{\mu_{0}} \nabla X \underline{B}\right)=0
$$


Equation (20) implies

$$
\sigma \underline{\text { UXB B }}-\frac{1}{\mu_{0}} \nabla X \underline{B}=-\nabla \Psi
$$

With only radial dependences, axial magnetic field, and radial flow, equation (21) becomes

$$
\begin{aligned}
-\sigma u B \hat{\phi}+\frac{1}{\mu_{0}} \frac{d B}{d r} \hat{\phi} & =-\frac{1}{r} \frac{\partial \Psi}{\partial \phi} \hat{\phi}-\hat{r} \frac{\partial \Psi}{\partial r}-\hat{z} \frac{\partial \Psi}{\partial r} . \\
& =-\frac{1}{r} \frac{d \Psi(\phi)}{d \phi} \hat{\phi} .
\end{aligned}
$$

Using equation (16) in equation (23) gives

$$
r \frac{d B}{d r}-\mu_{0} \sigma K_{2} B=-\mu_{0} \frac{d \Psi}{d \phi}
$$

We can see from equation (24) that $d \Psi / d \phi$ is a constant, since the left hand side is not a function of $\phi$. Equation (24) can be integrated with respect to $r$ by making the substitution

$$
\begin{aligned}
w & =\ln r \\
\frac{d}{d w} & =r \frac{d}{d r} .
\end{aligned}
$$

With this substitution in equation (24) and multiplying by $\exp \left(-\mu_{0} \sigma K_{2} W\right)$, we obtain

$$
e^{-\mu_{0} \sigma K_{2} W} \frac{d B}{d W}-\mu_{0} \sigma K_{2} B e^{-\mu_{0} \sigma K_{2} W}=-\mu_{0} \frac{d \Psi}{d \phi} e^{-\mu_{0} \sigma K_{2} W}
$$

or

$$
\frac{d}{d W}\left(B e^{-\mu_{0} \sigma K_{2} W}\right) \quad=\frac{1}{\sigma K_{2}} \frac{d}{d W}\left(e^{-\mu_{0} \sigma K_{2} W}\right) \frac{d \Psi}{d \phi} .
$$

Integrating yields

$$
B e^{-\mu_{0} \sigma K_{2} W}-B_{1} e^{-\mu_{0} \sigma K_{2} W_{1}}=\frac{1}{\sigma K_{2}} \frac{d \Psi}{d \phi}\left(e^{-\mu_{0} \sigma K_{2} W}-e^{-\mu_{0} \sigma K_{2} W_{1}}\right)
$$

or

$$
B=B_{1}\left(\frac{r}{r_{1}}\right)^{\mu_{0} \sigma u_{1} r_{1}}+\frac{1}{\sigma K_{2}} \frac{d \Psi}{d \phi}\left(1-\left(\frac{r}{r_{1}}\right)^{\mu_{0} \sigma u_{1} r_{1}}\right)
$$




$$
=\left(B_{1}-K_{3}\right)\left(\frac{r}{r_{1}}\right)^{\mu_{0} \sigma u_{1} r_{1}}+K_{3} .
$$

We have set $\left(\sigma K_{2}\right)^{-1} d \Psi / d \phi$ to the constant $K_{3}$.

Using equation (5) and equation (31) yields

$$
\mu_{0} \underline{J}=-\hat{\phi}\left(B_{1}-K_{3}\right) \mu_{0} \sigma u_{1}\left(\frac{r}{r_{1}}\right)\left(\mu_{0} \sigma u_{1} r_{1}-1\right)
$$

while

$$
\begin{aligned}
\underline{u} \underline{B}= & u \hat{r} \times B \hat{z} \\
& =-\hat{\phi}\left(B_{1}-K_{3}\right) u_{1}\left(\frac{r}{r_{1}}\right)^{\left(u_{0} \sigma u_{1} r_{1}-1\right)}-\hat{\phi} u_{1} r_{1} K_{3} / r .
\end{aligned}
$$

The electric field can be calculated using equation (32) and equation from

$$
\begin{aligned}
\underline{E} & =\frac{\underline{J}}{\sigma}-\underline{u X B} \underline{B} \\
& =\hat{\phi} u_{1} r_{1} k_{3} / r .
\end{aligned}
$$

Consider a circular path for which $\underline{\mathrm{d} \ell}=\hat{\phi} \mathrm{rd} \phi$ bounding a surface in a constant $Z$ plane. Applying equation (4), we obtain

$$
\begin{aligned}
\iint \nabla X \underline{E} \cdot \underline{d s} & =\oint \underline{E} \bullet \underline{d \ell} \\
& =\hat{\phi}\left(u_{7} r_{7} K_{3} / r\right) \cdot \hat{\phi} r d \phi \\
& =2 \pi u_{7} r_{7} K_{3} \\
& =0 .
\end{aligned}
$$

Hence $K_{3}$ is zero in steady state.

We can collect and summarize our results as:

$$
\begin{aligned}
& \underline{J}=-\hat{\phi} B_{1} \sigma u_{1}\left(\frac{r}{r_{1}}\right)\left(\mu_{0} \sigma u_{1} r_{1}-1\right) \\
& \underline{B}=B_{1}\left(\frac{r}{r_{1}}\right)^{\mu_{0} \sigma u_{1} r_{1}}
\end{aligned}
$$




$$
p=p_{1}+\frac{B_{1}^{2}}{2 \mu_{0}}\left(1-\left(\frac{r}{r_{1}}\right)^{2 \mu_{0} \sigma u_{1} r_{1}}\right)+\frac{1}{2} \rho u_{1}^{2}\left(1-\frac{r_{1}^{2}}{r^{2}}\right)
$$

Note the conspicuous role in equations (40-42) of the magnetic Reynolds number

$$
R_{m}=\mu_{0} \sigma\left|u_{1}\right| r_{1} .
$$

